McGill/94-48

hep-th/9411018

\title{
Gravity's Rainbow: Limits for the applicability of the equivalence principle
}

\author{
René Lafrance申 and Robert C. Myers各 \\ Department of Physics, McGill University \\ Montréal, Québec, Canada H3A 2T8
}

\begin{abstract}
Limits for the applicability of the equivalence principle are considered in the context of low-energy effective field theories. In particular, we find a class of higherderivative interactions for the gravitational and electromagnetic fields which produce dispersive photon propagation. The latter is illustrated by calculating the energydependent contribution to the deflection of light rays.
\end{abstract}

PACS numbers: $04.40 . \mathrm{Nr}, 11.25 . \mathrm{Mj}$

\footnotetext{
${ }^{1}$ lafrance@physics.mcgill.ca

${ }^{2}$ rcm@physics.mcgill.ca
} 


\section{Introduction}

The deflection of light by a gravitational field was one of the most striking predictions of general relativity. The observation of this effect was also one of the earliest experimental verifications of Einstein's theory[1]. The topic has presently matured to the stage where gravitational lensing is applied by astronomers for a variety of purposes [2], and in particular, it is providing an exciting new probe of the dark matter[3].

By the equivalence principle, photons fall freely along light-like geodesics in curved spacetime, and all photons are deflected in a gravitational potential by the same angle independently of their energy or their polarization. Things change when one goes beyond the standard minimal coupling of the electromagnetic and gravitational fields. For example in quantum electrodynamics, virtual electron loops will induce curvature couplings in the effective action of low-frequency photons [4, 5. In such a case, photon propagation can be influenced by tidal effects coming from local spacetime curvature. Using the one-loop effective action for QED, Drummonds and Hathrell [5] studied the propagation of photons in Schwarzschild, Robertson-Walker and gravitational-wave spacetimes. Apart from the Robertson-Walker background where the curvature is spatially isotropic, they found that the propagation of photons was polarization-dependent (gravitational birefringence). In particular, they considered light rays following transverse orbits in the Schwarzschild background, and found a polarization-dependent deflection angle. Similarly, gravitational birefringence was also found to occur in Reissner-Norström spacetimes [6].

In the context of string theory, Mende [7] also observed limits for the applicability of the equivalence principle. Since strings are extended objects, their propagation also feels tidal effects arising from the curvature of spacetime. Mende argued that this would imply energy-dependent deflection of light. Specifically, for photons following transverse orbits passing a spherically symmetric mass, the deflection angle would have a contribution proportional to the square of the photon energy. Further Mende argued that such a result would be a clear signature of string theory. We will show that such energy-dependent light scattering can also be produced within the context of a low-energy effective action for the electromagnetic field in curved spacetime [8].

The paper is organized as follows: In section 2, we establish the framework of our calculations. We review the geometric optics approximation, and illustrate how higherderivative interactions can modify the photon trajectories. The form of interactions which can lead to photon propagation with dispersion is also established. In section 3 , we consider various eight-derivative interactions, and show that they produce dispersive effects, such as energy-dependent deflection of light. Finally, section 4 presents a discussion of our results. Throughout the paper, we employ the conventions of ref. [9], and we use units where $\hbar=c=G=1$. 


\section{Geometric Optics Approximation}

Here, we will give a brief review of the geometric optics approximation - for more details, see ref. [9] and the references therein. We begin with the Maxwell action,

$$
I_{0}=-\frac{1}{4} \int d^{4} x \sqrt{-g} F_{\mu \nu} F^{\mu \nu}
$$

where $F_{\mu \nu}=\partial_{\mu} A_{\nu}-\partial_{\nu} A_{\mu}$. We impose the Lorentz gauge condition

$$
\nabla^{\mu} A_{\mu}=0,
$$

in which case the equation of motion may be written as

$$
\nabla^{\mu} F_{\mu \nu}=\nabla^{2} A_{\nu}-R_{\nu}{ }^{\mu} A_{\mu}=0 .
$$

In the geometric optics approximation, one assumes that the electromagnetic waves have a wavelength which is much smaller than the radius of curvature of the background geometry, or the scale for variations of the amplitude of the wave front. The gauge potential is written as

$$
A_{\mu}=a_{\mu} \exp [i \theta]
$$

where $a_{\mu}$ is a slowly varying amplitude, and $\theta$, a rapidly varying phase. (It is understood that one takes the real part of the right hand side of eq. (田). The wave vector $k_{\mu}=\nabla_{\mu} \theta$ is normal to surfaces of constant phase, and light rays are defined by $\frac{d x^{\mu}}{d s}=k^{\mu}$. When this ansatz (4) is inserted into the equation of motion and the gauge condition, the leading terms come from the derivatives of the phase. Thus eq. (3) yields

$$
k^{\mu} k_{\mu}=0
$$

while eq. (2) produces

$$
k^{\mu} a_{\mu}=0
$$

at leading order - i.e., light rays are null geodesics, and the polarization is orthogonal to the wave vector. At next order, eq. (3) also yields a propagation equation for the amplitude, $k^{\mu} \nabla_{\mu} a_{\nu}+\frac{1}{2}\left(\nabla_{\mu} k^{\mu}\right) a_{\nu}=0$. One can continue with a systematic expansion of post-geometricoptics corrections, which would be necessary to realize the full wave-like character of the solutions - e.g., diffraction or interference. For our purposes, though, we restrict our attention to the leading-order geometric-optics equation. It is also assumed above and in the following that the perturbation of the background metric by the electromagnetic waves is negligible.

For our purposes, an important feature of the above leading-order equations is that they are invariant under a constant scaling of the wave vector, $k_{\mu} \rightarrow \alpha k_{\mu}$. Thus to this

order, all solutions will behave identically independent of the frequency of the photons. Thus physical effects, such as the bending of light rays, are independent of the photon energy, E. Note that this scale invariance does not hold for the post-geometric-optics 
corrections, which do then yield frequency or energy dependent results. In the problem of light ray deflection, these corrections, which produce contributions proportional to $1 / E$, signify diffractive (i.e., wave-like) effects. In contrast, the effects, which we determine in the following section, are proportional to $E^{2}$ and modify the light rays in the leading-order approximation.

Now we will consider modifying the Maxwell action by adding higher-derivative interactions, as may occur in an effective field theory. Assuming that the former are quadratic in the electromagnetic field strength, they will generically modify the light-cone condition (5) within the geometric optics approximation. Throughout the following, we still impose the Lorentz gauge condition (2), and so eq. (6) remains unchanged. To illustrate the modifications produced by higher-derivative interactions, consider

$$
I_{1}=-\frac{\alpha \lambda_{e}^{2}}{360 \pi} \int d^{4} x \sqrt{-g} R_{\mu \nu \sigma \tau} F^{\mu \nu} F^{\sigma \tau}
$$

which arises as an interaction in the one-loop effective action for QED [4, 5]. Here, $\lambda_{e}$ is the Compton wavelength of the electron, and $\alpha$ is the fine structure constant. There are other terms involving $R_{\mu \nu}$ and $R$, but they will be unimportant when considering photon propagation in a background spacetime which is a solution of the vacuum Einstein equations. Combining eq.'s (11) and (7), the equation of motion becomes

$$
\nabla^{\mu} F_{\mu \nu}+\frac{\alpha \lambda_{e}^{2}}{90 \pi}\left(R_{\mu \nu \sigma \tau} \nabla^{\mu}+2 \nabla_{\sigma} R_{\tau \nu}\right) F^{\sigma \tau}=0
$$

where the Bianchi identity for the Riemann tensor has been applied in the last term. Inserting the geometric optics ansatz (4) and applying the Lorentz gauge condition, we find

$$
k^{2} a_{\nu}+\frac{\alpha \lambda_{e}^{2}}{45 \pi} R_{\mu \nu \sigma \tau} k^{\mu} k^{\sigma} a^{\tau}=0
$$

as the new leading order equation (where $k^{2}=k^{\mu} k_{\mu}$ ). While the light-cone has been modified away from $k^{2}=0$, the new equation is still invariant when the wave-vector is scaled. Thus the resulting photon trajectories are still frequency independent. The coupling to the Riemann tensor in eq. (9) does break local Lorentz invariance, and this equation has been found to produce polarization-dependent light propagation [5].

To have dispersive results then, the leading-order equation cannot be invariant under scaling of $k_{\mu}$. Thus one must consider interactions with more derivatives, and in particular, the interactions must contribute to the electromagnetic equations of motion with more derivatives of the gauge potential. With this in mind, a natural extension of eq. (17) is then

$$
I_{2}=-\frac{\beta \lambda^{4}}{4} \int d^{4} x \sqrt{-g} R_{\mu \nu \sigma \tau} \nabla_{\rho} F^{\mu \nu} \nabla^{\rho} F^{\sigma \tau}
$$

where $\beta$ is a dimensionless coupling constant, and $\lambda$ is the (length) scale associated with the effective interaction. Combined with the Maxwell action (11), this new term leads to an equation of motion

$$
\nabla_{\mu} F^{\mu \nu}-\beta \lambda^{4} \nabla_{\mu} \nabla_{\rho}\left(R^{\mu \nu \sigma \tau} \nabla^{\rho} F_{\sigma \tau}\right)=0
$$


and in the leading order of the geometric optics approximation, one finds

$$
k^{2}\left(a_{\nu}+2 \beta \lambda^{4} R_{\mu \nu \sigma \tau} k^{\mu} k^{\sigma} a^{\tau}\right)=0 .
$$

We will assume that the effective action is constructed perturbatively in the coupling $\beta$. Within in such a framework, even though eq. (12) is not invariant under scaling of $k_{\mu}$, the light-cone condition remains $k^{2}=0$. The second factor in brackets would define spurious characteristics which are nonperturbative in $\beta$. Alternatively, one may say that perturbatively we wish to calculate to modification of eq. (5) at order $\beta$, and so expect to find $k^{2}=O(\beta)$ in general. Substituting the latter into the term proportional to $\beta$ in eq. (12), we in fact have $k^{2}=O\left(\beta^{2}\right)$ and so there is no perturbation of the light-cone to the order which we are calculating.

There are other six-derivative interactions similar to $I_{2}$ where the indices are contracted in different ways, but in the equations of motion, the higher-derivative terms are proportional to $k^{2}$ again, or to the gauge condition $k \cdot a$ which vanishes. Thus we found that there are no six-derivative interactions which will produce a dispersive light-cone condition. To obtain an energy dependent result, one needs to consider interactions with both more derivatives and more background curvatures in order to avoid the above contractions.

\section{Dispersive interactions}

From the last section then, we have learned that in order to produce a dispersive modification of the light-cone condition, we need an interaction which is quadratic in the field strength, has four derivatives of the gauge potential, and has more than one background curvature or derivatives of the background curvature. In this section following these criteria, we construct a number of eight-derivative interactions, and show that they lead to energy-dependent photon propagation. We begin with a simple extension of eq. (10), where a second curvature tensor is introduced.

$$
I_{3}=-\frac{\beta \lambda^{6}}{4} \int d^{4} x \sqrt{-g} R^{\kappa \alpha \beta \gamma} R_{\kappa}{ }^{\rho \mu \nu} \nabla_{\alpha} F_{\beta \gamma} \nabla_{\rho} F_{\mu \nu}
$$

where $\beta$ and $\lambda$ are the coupling and scale, as above. The equation of motion for the electromagnetic field becomes

$$
\nabla_{\mu} F^{\mu \nu}-\beta \lambda^{6} \nabla_{\mu} \nabla_{\rho}\left(R^{\kappa \alpha \beta \gamma} R_{\kappa}{ }^{\rho \mu \nu} \nabla_{\alpha} F_{\beta \gamma}\right)=0 .
$$

This (along with the gauge constraint (6)) yield in the geometric optics approximation,

$$
k^{2} a^{\nu}+2 \beta \lambda^{6} R^{\kappa \alpha \beta \gamma} R_{\kappa}{ }^{\mu \nu \nu} k_{\alpha} k_{\beta} k_{\rho} k_{\mu} a_{\gamma}=0 .
$$

As desired, these equations are not invariant under scaling of the wave vector, and the higher order term is not proportional to the original light-cone condition, $k^{2}$. Hence these 
equations produce dispersion, and should lead to energy-dependent light deflection in a gravitational potential.

To explicitly display such dispersive light scattering, we now turn to the specific background of the Schwarzschild metric in standard coordinates [9]

$$
d s^{2}=-\left(1-\frac{2 M}{r}\right) d t^{2}+\left(1-\frac{2 M}{r}\right)^{-1} d r^{2}+r^{2}\left(d \theta^{2}+\sin ^{2} \theta d \phi^{2}\right)
$$

We introduce the vierbein $e^{a}{ }_{\mu}(a=0,1,2,3)$

$$
e^{a}{ }_{\mu}=\operatorname{diag}(U, 1 / U, r, r \sin \theta)
$$

satisfying $g_{\mu \nu}=\eta_{a b} e^{a}{ }_{\mu} e^{b}{ }_{\nu}$ where $U=\left(1-\frac{2 M}{r}\right)^{1 / 2}$ and $\eta_{a b}=\operatorname{diag}(-1,1,1,1)$. The Riemann tensor is conveniently expressed as

$$
R^{\mu \nu \sigma \tau}=-\frac{M}{r^{3}}\left[g^{\mu \sigma} g^{\nu \tau}-g^{\mu \tau} g^{\nu \sigma}+3 U_{01}^{\mu \nu} U_{01}^{\sigma \tau}-3 U_{23}^{\mu \nu} U_{23}^{\sigma \tau}\right]
$$

using the bivectors

$$
U_{a b}^{\mu \nu}=e_{a}^{\mu} e_{b}^{\nu}-e_{a}^{\nu} e_{b}^{\mu} .
$$

In the Schwarzschild background, the leading order equations (15) may be written as:

$$
\left[k^{2} \delta_{b}^{a}+\zeta X_{b}^{a}\right] a^{b}=0
$$

where $\zeta=2 \beta \lambda^{6} \frac{M^{2}}{r^{6}}$ and using $k_{a}=e_{a}{ }^{\mu} k_{\mu}$

$$
X_{b}^{a}=\left(\begin{array}{cccc}
-k_{1}^{2} A+k^{2} m^{2} & -k_{0} k_{1} A & k_{0} k_{2} k^{2} & k_{0} k_{3} k^{2} \\
k_{0} k_{1} A & k_{0}^{2} A+k^{2} m^{2} & -k_{1} k_{2} k^{2} & -k_{1} k_{3} k^{2} \\
-k_{0} k_{2} k^{2} & -k_{1} k_{2} k^{2} & k_{3}^{2} B-k^{2} l^{2} & -k_{2} k_{3} B \\
-k_{0} k_{3} k^{2} & -k_{1} k_{3} k^{2} & -k_{2} k_{3} B & k_{2}^{2} B-k^{2} l^{2}
\end{array}\right)
$$

where we have defined $l^{2} \equiv k_{0}^{2}-k_{1}^{2}, m^{2} \equiv k_{2}^{2}+k_{3}^{2}, A \equiv\left(4 l^{2}+5 m^{2}\right)$, and $B \equiv\left(5 l^{2}+4 m^{2}\right)$. Note that $k^{2}=-l^{2}+m^{2}$. To have a non-trivial solution of (19), the determinant of the matrix in square brackets must vanish.

$$
k^{2}\left[k^{2}\left(1+\zeta k^{2}\right)\right]\left[k^{2}+4 \zeta l^{4}+4 \zeta l^{2} m^{2}+\zeta m^{4}\right]\left[k^{2}+4 \zeta m^{4}+4 \zeta m^{2} l^{2}+\zeta l^{4}\right]=0
$$

Here the four factors actually correspond to the eigenvalues of the matrix, and the polarization associated with a given light-cone condition will be given by the corresponding eigenvector. To simplify the analysis, consider photon trajectories in the plane $\theta=\pi / 2$, which corresponds to $k_{2}=0$ (or $k_{\theta}=0$ ), in which case $m^{2}=k_{3}^{2}$.

1. For the eigenvalue, $k^{2}$, the polarization eigenvector is $a^{b}=\left(-k_{0}, k_{1}, 0, k_{3}\right)=k^{b}$. This is, of course, the expected unphysical polarization which associated with the leading order gauge invariance, $a_{\mu} \rightarrow a_{\mu}+k_{\mu}-i . e$, since the gauge condition (6) was not used in the $O(\beta)$ term in eq. (15), one must have $X_{{ }_{b}}^{a} k^{b}=0$. 
2. The polarization corresponding to the second eigenvalue, $k^{2}\left(1+\zeta k^{2}\right)$, is $a^{b}=\left(-k_{0} k_{3}, k_{1} k_{3}, 0, k_{0}{ }^{2}-k_{1}^{2}\right)=k_{3} k^{b}-\left(0,0,0, k^{2}\right)$. Now the vanishing of the eigenvalue yields $k^{2}=0$ or $k^{2}=-1 / \zeta$. The latter result is nonperturbative in the coupling, and so the only relevant result is the original light-cone $k^{2}=0$. Note that in this case the polarization is degenerate with that in case (i), and so it is also unphysical. Further note that $k \cdot a=0$ is satisfied here independent of whether or not one imposes $k^{2}=0$.

3. For the third eigenvalue, one finds a generalized light-cone condition: $k^{2}+\zeta\left(4 l^{4}+\right.$ $\left.4 l^{2} m^{2}+m^{4}\right)=0$. The corresponding polarization is $a^{b}=\left(-k_{1}, k_{0}, 0,0\right)$, which also satisfies the Lorentz gauge condition. Thus this case corresponds to photons with a radial polarization.

4. The final eigenvalue yields $k^{2}+\zeta\left(4 m^{4}+4 m^{2} l^{2}+l^{4}\right)=0$ for a polarization $a^{b}=$ $(0,0,1,0)$ along the $z$-axis - i.e., orthogonal to the $\theta=\pi / 2$ plane. Note that the Lorentz gauge condition is also satisfied here.

To leading order, one has $k^{2}=O(\zeta)$ in either of the last two cases. Hence if one applies $k^{2}=0$ in the $O(\zeta)$ contributions, one has $l^{2}=m^{2}$ and so (iii) and (iv) both produce the same perturbed light-cone condition $k^{2}+9 \zeta l^{4}=0$. Thus to the order of accuracy which we are calculating there is no gravitational birefringence here - i.e., all physical polarizations will follow the same trajectories. One may also note that for radial motion $\left(k_{3}=0\right)$, one has $l^{2}=m^{2}=0$ in the $O(\zeta)$ contribution, and this light-cone reduces to the usual $k^{2}=0$.

Now we calculate the modifications to the deflection angle for photon trajectories which begin at $y \rightarrow-\infty$ and approach the central mass parallel to the $y$-axis in the $x$ - $y$ plane with an impact parameter $b$ [9, 10]. In the Schwarzschild background using standard coordinates, the generalized light-cone condition is

$$
-\left(1-\frac{2 M}{r}\right)\left(1-9 \zeta l^{2}\right) k^{t} k^{t}+\left(1-\frac{2 M}{r}\right)^{-1}\left(1-9 \zeta l^{2}\right) k^{r} k^{r}+r^{2} k^{\theta} k^{\theta}+r^{2} \sin ^{2} \theta k^{\phi} k^{\phi}=0 .
$$

Working perturbatively in $\zeta$, it is sufficient to use the classical value for $l^{2}=k_{0} k_{0}-k_{1} k_{1}=$ $E^{2} b^{2} / r^{2}$ above, where $E$ is the energy of the photon and $b$, the impact parameter [9]. Following Ref.[5], the simplest way to determine the deflection angle from the light-cone condition is to consider the wave vector $k_{\mu}$ as a null vector in an effective metric

$$
d s^{2}=-B(r) d t^{2}+A(r) d r^{2}+r^{2} d \theta^{2}+r^{2} \sin ^{2} \theta d \phi^{2}
$$

with

$$
\begin{aligned}
& A(r)=\left(1-\frac{2 M}{r}\right)^{-1}\left(1-\frac{9 \zeta E^{2} b^{2}}{r^{2}}\right) \\
& B(r)=\left(1-\frac{2 M}{r}\right)\left(1-\frac{9 \zeta E^{2} b^{2}}{r^{2}}\right) .
\end{aligned}
$$


The deflection angle is then given by [10]

$$
\Delta \phi+\pi=2 \int_{r_{0}}^{\infty} \frac{d r}{r}\left[\frac{A(r)}{\frac{r^{2}}{r_{0}^{2}} \frac{B\left(r_{0}\right)}{B(r)}-1}\right]^{1 / 2}
$$

where $r_{0}$ is the distance of closest approach (at which $k^{r}$ changes sign). For the unperturbed Schwarzschild metric, eq. (23) yields $\Delta \phi=4 M / r_{0}$ [10]. Expanding $A(r)$ and $B(r)$ in power of $\zeta$ and keeping only the linear terms, one obtains an integral for $\delta \Delta \phi$

$$
\delta \Delta \phi=\int_{r_{0}}^{\infty} \frac{d r}{r}\left[\frac{\delta A(r)}{\left(\frac{r^{2}}{r_{0}^{2}}-1\right)^{1 / 2}}-\frac{r^{2}}{r_{0}^{2}} \frac{\delta B\left(r_{0}\right)-\delta B(r)}{\left(\frac{r^{2}}{r_{0}^{2}}-1\right)^{3 / 2}}\right] .
$$

Inserting $\delta A(r)=\delta B(r)=-9 \zeta E^{2} b^{2} / r^{6}$, one obtains for both polarizations

$$
\delta \Delta \phi=\frac{2205 \pi}{128} \frac{\beta \lambda^{6} M^{2} E^{2}}{r_{0}^{6}} .
$$

Note that both this result and $\Delta \phi=4 M / r_{0}$ are leading order expressions, which are corrected by terms which are higher order in $M / r_{0}$.

A second interaction which produces similar dispersive results is

$$
I_{4}=-\frac{\beta \lambda^{6}}{4} \int d^{4} x \sqrt{-g} R^{\alpha \kappa \beta \lambda} R_{\alpha \mu \beta \nu} \nabla_{\kappa} F_{\lambda \rho} \nabla^{\mu} F^{\nu \rho}
$$

In this case, the equation of motion for the electromagnetic field becomes

$$
\nabla_{\mu} F^{\mu \nu}-\beta \lambda^{6} \nabla_{\rho} \nabla_{\mu}\left(R_{\alpha \kappa \beta \lambda} R^{\alpha \mu \beta[\rho \mid} \nabla^{\kappa} F^{\lambda \mid \nu]}\right)=0
$$

where the square brackets indicate that the expression is antisymmetrized in $\rho$ and $\nu$ with a factor of $1 / 2$. Inserting the geometric ansatz (4) and applying the gauge constraint (6) produces the leading order equation

$$
k^{2} a^{\nu}+\beta \lambda^{6} R_{\alpha \kappa \beta \lambda} R^{\alpha \mu \beta \rho} k_{\rho} k_{\mu} k^{\kappa} k^{[\lambda} a^{\nu]}=0 .
$$

One may now follow the procedure used above to determine the modified light-cone condition for photons in a Schwarzschild background. A simpler approach for this specific case yields a general light-cone condition - namely, contract eq. (27) with $a_{\nu}$. Upon applying the gauge condition (6) and extracting a factor of $a^{2}$, one obtains

$$
k^{2}+\frac{\beta \lambda^{6}}{2} R_{\alpha \mu \beta \rho} R^{\alpha \kappa \beta \lambda} k_{\lambda} k_{\kappa} k^{\mu} k^{\rho}=0
$$

Thus we have a general light-cone condition which describes all polarizations, and so there will be no gravitational birefringence in any background. (Note that the unphysical polarizations do not satisfy $k^{2}=0$ in this case, because in arriving at eq. (28) we have used 
the gauge constraint in the $O(\beta)$ terms). In the Schwarzschild background, this light-cone becomes

$$
k^{2}+\frac{\zeta}{4}\left[9 l^{4}+9 m^{4}-3 k^{4}\right]
$$

where as above, we use $\zeta=\frac{2 \beta \lambda^{6} M^{2}}{r^{6}}, l^{2}=k_{0}^{2}-k_{1}^{2}$, and $m^{2}=k_{2}^{2}+k_{3}^{2}$. To leading order, $k^{2}=\mathcal{O}(\beta)$ and $m^{2}=l^{2}+\mathcal{O}(\beta)$ and so eq. (29) reduces to $k^{2}+\frac{9}{2} \zeta l^{4}$. Hence up to a factor of two, we have recovered precisely the same dispersive light-cone as in the analysis of the interaction $I_{3}$ (for the physical polarizations). The modification to the deflection of light is therefore one half the angle obtained in eq. (24).

A final eight-derivative interaction which produces dispersive light propagation was found by extending eq. (10) by introducing extra background derivatives, rather than an extra curvature tensor

$$
I_{5}=-\frac{\beta \lambda^{6}}{4} \int d^{4} x \sqrt{-g} \nabla_{(\rho} \nabla_{\sigma)} R_{\alpha \beta \mu \nu} \nabla^{\rho} F^{\alpha \beta} \nabla^{\sigma} F^{\mu \nu}
$$

where $\nabla_{(\rho} \nabla_{\sigma)}=1 / 2\left(\nabla_{\rho} \nabla_{\sigma}+\nabla_{\sigma} \nabla_{\rho}\right)$. After adding $I_{5}$ to the Maxwell action (四), the equation of motion for the electromagnetic field becomes

$$
\nabla_{\mu} F^{\mu \nu}-\beta \lambda^{6} \nabla_{\mu} \nabla_{\sigma}\left(\nabla^{(\sigma} \nabla^{\rho)} R^{\alpha \beta \mu \nu} \nabla_{\rho} F_{\alpha \beta}\right)=0
$$

We then insert the geometric optics ansatz (4) to obtain:

$$
k^{2} a^{\nu}+2 \beta \lambda^{6} \nabla^{\sigma} \nabla^{\rho} R^{\alpha \beta \mu \nu} k_{\sigma} k_{\rho} k_{\mu} k_{\alpha} a_{\beta}=0
$$

To calculate the light-cone conditions in the Schwarzschild background, we followed the same method that was used in the analysis of $I_{3}$ above. The leading order equations may be written

$$
\left[k^{2} \delta_{b}^{a}+\eta \widehat{X}_{b}^{a}\right] a^{b}=0
$$

where $\eta=60 \beta \lambda^{6} M / r^{5}$. The matrix $\widehat{X}^{a}{ }_{b}$ is a lengthy expression involving $k_{a}$, which we will not display explicitly here. As before, the modified light-cone conditions are given by the vanishing of the eigenvalues of the matrix in square brackets, and the corresponding eigenvectors give the associated polarizations. If we consider photon trajectories in the plane $\theta=\pi / 2$ and we apply $k^{2}=\mathcal{O}(\beta)$ in $\widehat{X}^{a}{ }_{b}$, we find:

1. $k^{2}=0$ for the two unphysical polarizations

2. $k^{2}+\eta C l^{2}=0$ for the radial polarization

3. $k^{2}-\eta C l^{2}=0$ for the polarization orthogonal to the plane of motion.

where

$$
C=\left(1-\frac{3 M}{r}\right) k_{0}^{2}-\left(7-\frac{15 M}{r}\right) k_{1}^{2}
$$


Since the light-cones for the radial and theta polarizations differ, this last case provides an example of gravitational birefringence. Calculating the deflection angle as above, we find an energy-dependent contribution

$$
\delta \Delta \phi= \pm 504 \frac{\beta \lambda^{6} M E^{2}}{r_{0}^{5}}
$$

where the plus sign corresponds to the radial polarization and the minus sign, to the theta polarization. Note that this result is one order lower in the $M / r_{0}$ expansion than the previous result (24). This reduction occurs since the present interaction (30) involves a single Riemann tensor, while the previous interactions have two curvature tensors.

\section{Discussion}

In this paper, we have found some explicit field theory interactions that produce dispersive photon propagation, in the context of an effective field theory where the Maxwell action is modified by higher-derivative terms. Such dispersion was not observed in earlier studies simply because the effective actions considered previously did not include sufficiently high numbers of derivatives. The final case also provides a new example of gravitational birefringence. One may ask whether there will be other eight-derivative interactions which will produce dispersion, and clearly the answer is yes. The three interactions that we have considered though are representatives of three classes of interactions, which produce the same leading order equations in the geometric optics approximation. It is not hard to verify that eq.'s (15), (28) and (31) are unique. For instance, there is only a single way to contract four wave-vectors and one polarization with a double derivative of the background curvature tensor, and this is the combination appearing in eq. (31). Thus,

$$
I_{5}^{\prime}=-\frac{\beta \lambda^{6}}{2} \int d^{4} x \sqrt{-g} \nabla_{(\rho} \nabla_{\sigma)} R_{\alpha \beta \mu \nu} \nabla^{\rho} F^{\sigma \alpha} \nabla^{\beta} F^{\mu \nu}
$$

leads to precisely eq. (31) as the leading order equations of motion. The two interactions, $I_{5}$ and $I_{5}^{\prime}$, differ by total derivatives, and also terms which do not contribute to these leading order contributions (i.e., they do not contribute to the dispersion).

In eq.'s (24) and (34), we have found contributions to the deflection angle of the light rays, which depends on the square of the photon energy. This behavior is the same as that found for string theory by Mende. One may ask then if interactions of the form discussed here appear amongst the higher dimension interactions included in the lowenergy effective string action. There are two alternative approaches to constructing these low-energy actions. First, it can be determined from the sigma model beta functions, which define the low energy string equations of motion [11, 12]. Unfortunately, sigma model calculations involving background metric and gauge fields have not been carried out to sufficient order to detect terms of the form suggested here. Alternatively, the higher-derivative terms in the low-energy action can be determined by requiring that the 
resulting field theory reproduce the string scattering amplitudes to the corresponding order in $\alpha^{\prime} p^{2}$ [11, 13], where $p$ here represents a typical momentum from the scattering process, and $\alpha^{\prime}=\lambda_{s t}^{2}$ is the string scale squared (essentially, this corresponds to the Planck scale squared). Interactions of the form (13), (25) or (30) would contribute to an scattering amplitude of two photons and two gravitons - the contribution of $I_{5}$ to a two photon and one graviton amplitude vanishes on-shell. A sufficiently detailed study of the low-energy effective action for heterotic string has been made to detect terms of the form discussed here [13, but unfortunately, one finds that these terms do not appear in this action. This suggests that Mende's dispersive effect, which should be universal to all string theories[7], must be produced by an interaction at an even higher order in the $\alpha^{\prime}$ expansion (or the expansion in numbers of derivatives) than considered in the present paper. So one would expect that the dependence on the radius of closest approach is even more dramatic than the $r_{0}^{-6}$ appearing in eq. (24). Additional Riemann tensors would also increase the power of the central mass appearing in the dispersive contribution to the deflection angle.

If one considers studies of low-energy string actions, there is one eight-derivative interaction which is in fact universal to all string theories 14

$$
I_{6}=\frac{\zeta(3) \alpha^{\prime 3}}{512 \pi} \int d^{D} x \sqrt{-g}\left(2 R_{\alpha \beta \rho \sigma} R_{\mu}^{\beta \rho}{ }_{\nu} R^{\alpha \gamma \eta \mu} R_{\gamma \eta}^{\nu}{ }^{\sigma}+R_{\alpha \beta \rho \sigma} R_{\mu \nu}{ }^{\rho \sigma} R^{\alpha \gamma \eta \mu} R_{\gamma \eta}^{\nu}{ }^{\beta}\right)
$$

where $\zeta(s)$ is the Riemann zeta function. We have also indicated that this effective action is in $D$ dimensions, since typically string theories are constructed for $D>4$. If the spacetime is then compactified down to four dimensions via a Kaluza-Klein ansatz [15, then new vector particles will appear in the effective theory arising from off-diagonal components of the metric, which mix the four-dimensional spacetime with the compact directions (e.g., $g_{\mu 5} \simeq A_{\mu}$ ). The $D$-dimensional Einstein action provides the standard Maxwell action (11) for these vectors upon compactification. Similarly one finds that upon compactification the above interaction yields interactions of the form of eq.'s (13) and (25) (e.g., using $\left.R_{5 \alpha \beta \mu} \simeq-\frac{1}{2} \nabla_{\alpha} F_{\beta \mu}+\ldots\right)$. Therefore the above string interaction produces dispersive propagation as described in our present analysis for these Kaluza-Klein vector fields. The latter, of course, correspond to particular modes in the string spectrum.

Finally, we consider the magnitude of the deflection angles that we have calculated. Ultimately, we expect that this dispersion would only be observable in very exotic circumstances, but to begin let us evaluate eq. (34) with solar parameters for which the leading order deflection angle of general relativity is $\Delta \phi=4 M / r_{0}=1^{\prime \prime} .75$ [9]. The length scale $\lambda$ is the microphysical scale associated with the processes that induce our effective interaction. Here, we will choose the interaction scale to correspond the Compton wavelength of the electron (i.e., $\lambda=\lambda_{e} \simeq 2.4 \times 10^{-12} \mathrm{~m}$ ) as it would be if eq. (30) arose as a higher order term in the derivative expansion of the one-loop effective action for QED - clearly the effect will be more suppressed if we chose a shorter length scale, e.g., the Planck scale in a string effective action. In this case, it is natural to choose the dimensionless coupling constant to be of the order of the fine structure constant (i.e., $\beta \simeq \alpha$ ). With these choices, the dispersive deflection angle for a radially polarized photon grazing over the limb of the 
$\operatorname{sun}\left(\right.$ i.e., $\left.r_{0} \simeq 7 \times 10^{8} \mathrm{~m}\right)$ is given by

$$
\frac{\delta \Delta \phi}{\Delta \phi} \simeq \frac{10^{-89}}{\lambda_{p h}^{2}}
$$

where $\lambda_{p h}$ is the wavelength of the photon measured in angstroms. So the visible spectrum ranging from four- to seven-thousand angstroms would be spread over an angle of about $6 \times 10^{-98}$ arcseconds. Clearly as such, gravity's rainbow would be unobservable.

Now we also wish to consider situations in which the dispersion would become more pronounced. If we are consider eq. (24) or (34) with $\lambda$ and $\beta$ fixed as above for the QED (i.e., $\lambda=\lambda_{e}$ and $\beta \simeq \alpha$ ), there are three options: increase the photon energy, decrease the radius of closest approach or increase the central mass. With any of these options, we are limited by the approximations entering into our calculations. The deflection of much higher energy photons is certainly greater, but one must remember that the applicability of the effective action limited to photon wavelengths greater than the interaction scale $\lambda$, which we are here considering to be the Compton wavelength of the electron. Thus one could only consider photons up to the X-ray portion of the spectrum. The deflection is also increased with a reduction in the radius of closest approach $r_{0}$. This radius would be minimized by considering a black hole for which one might achieve $r_{0} \simeq M$. Such a scenario, though, runs into conflict with another approximation made in our scattering angle calculations, namely $M / r_{0} \ll 1$. In principle, one could carry out those calculations in more detail if one wished to consider $M / r_{0} \simeq 1$. With this choice then, one would actually want to decrease, rather than increase, the mass, $M$. Here the limitation is the validity of the geometric optics approximation, which requires that the photon wavelength be much smaller than the radius of curvature of the spacetime geometry. In a Schwarzschild geometry then, one demands that $\lambda_{p h}^{2}<r_{0}^{3} / M \simeq M^{2}$. Thus at least $M$ must be greater than $\lambda_{e}$, which was a lower bound on the photon wavelength. Certainly, one could imagine then that dramatic dispersion would be produced for X-rays by a black hole of $M \simeq 10^{18} \mathrm{~g}$, for which the gravitational radius would be of the order of the Compton wavelength of electron. It seems, though, that such an object (with $M \simeq 10^{-15} M_{\odot}$ ) and the dispersed X-rays are unlikely to be observed. It may also be interesting though to consider photon propagation beyond the geometric optics approximation. It may be that effective interactions of lower dimension than considered in section 3 could produce dispersion in situations with large and rapidly varying curvatures, as could possibly be created by gravitational collapse. In conclusion, while the dispersive photon propagation appearing in the present analysis in

principle presents a violation of the equivalence principle, it appears to be beyond the practical limits of observations.

\section{Acknowledgments}

This research was supported by NSERC of Canada and Fonds FCAR du Québec. We would like to thank Cliff Burgess, Paul Mende and Joe Polchinski for useful conversations. 


\section{References}

[1] F.W. Dyson, A.S.E. Eddington and C.R. Davidson, Phil. Trans. Roy. Soc. A 220, 291 (1920).

[2] See for example: P. Schneider, J. Ehlers and E.E. Falco, Gravitational Lenses (Springer Verlag, Berlin, 1992); R.D. Blanford and R. Narayan, Ann. Rev. Astron. Astrophys. 30, 311 (1992).

[3] C. Alcock et al., Nature 365, 621 (1993); E. Aubourg et al., Nature 365, 623 (1993); A. Udalski et al. , Act. Astro. 43, 289 (1993); A. Udalski et al. , ApJ 426, L69 (1994).

[4] F.A. Berends and R. Gastmans, Ann. Phys. (N.Y.) 98, 225 (1976) ; K.A. Milton, Phys. Rev. D15, 2149 (1977).

[5] I.T. Drummonds and S.J. Hathrell, Phys. Rev. D22, 343 (1980).

[6] R.D. Daniels and G.M. Shore, Nucl. Phys. B425, 634 (1994).

[7] P.F. Mende, in String Quantum Gravity and Physics at the Planck Scale, Proceedings of the Erice Workshop, 1992, edited by N. Sanchez (World Scientific, Singapour, 1993).

[8] Berends and Gastmans[国] reported to find energy-dependent light deflection within the context of the one-loop effective action from QED, but this was a erroneous result, as described by Drummonds and Hathrell[5].

[9] C.W. Misner, K.S. Thorne and J.A. Wheeler, Gravitation (Freeman, San Francisco, 1973).

[10] S. Weinberg, Gravitation and Cosmology (Wiley, New-York, 1972).

[11] See, e.g., M. Green, J. Schwarz and E. Witten, Superstring Theory (Cambridge University Press, Cambridge, 1987).

[12] C.G. Callan, D. Friedan, E.J. Martinec and M.J. Perry, Nucl. Phys. B262, 593 (1985).

[13] D.J. Gross and J.H. Sloan, Nucl. Phys. B291, 41 (1987).

[14] D.J. Gross and E. Witten, Nucl. Phys. B277, 1 (1986); M.T. Grisaru, A.E.M. van de Ven and D. Zanon, Phys. Lett. B173, 423 (1986); Nucl. Phys. B277, 388 (1986); Nucl. Phys. B277, 409 (1986); M.T. Grisaru and D. Zanon, Phys. Lett. B177, 347 (1986).

[15] Th. Kaluza, Sitz. Preuss. Akad. Wiss. K1, 966 (1921); O. Klein, Z. Phys. 37, 895 (1926). 